\title{
Heart rate recovery and diastolic blood pressure ratio on the treadmill test predict an induction and recurrence of vasovagal syncope
}

\author{
Yu Jeong Choi ${ }^{1}$, Ki-Woon Kang ${ }^{1}$, Sang Hyun Jang ${ }^{2}$, Jae Guk Kim², Soo Joo Lee ${ }^{2}$, and Kyung Tea Jung ${ }^{1}$
}

Divisions of ${ }^{\mathrm{C} C a r d i o l o g y}$ and ${ }^{2}$ Neurology, Eulji University Hospital, Daejeon, Korea
Background/Aims: The induction and recurrence of syncope is a concerning situation that could be unpredicted in the vasovagal syncope (VVS). We investigated a simple predictor for the induced and recurrent VVS during Head-Up table-tilt Test (HUT) and clinically follow-up.

Methods: The 143 consecutive patients with VVS (age $31 \pm 19$ years, 33 male) who referred by a cardiologist or neurologist and had undergone an echocardiogram, HUT, and a treadmill exercise test (TMT) were recruited and clinically follow-up. Patients were divided into two groups based on the result of HUT and TMT. The data was analyzed and compared between VVS patients and control 141 patients without VVS who were enrolled in the same study period (age $40 \pm 5$ years, 117 male).

Results: The heart rate recovery (HRR), recovery systolic blood pressure (RecSBP), recovery diastolic blood pressure (RecDBP), HRR/RecSBP and HRR/RecDBP were significantly different between controls and VVS during the TMT. Within VVS, even if, baseline characteristics were similar between negative and positive HUT $(\mathrm{n}=92$ vs. $\mathrm{n}=51) . \mathrm{HRR}(31 \pm 10$ vs. $35 \pm 10), \mathrm{HRR} / \operatorname{RecSBP}(0.24 \pm 0.09$ vs. $0.28 \pm 0.09)$ and HRR/RecDBP (0.49 \pm 0.18 vs. $0.58 \pm 0.19)$ were significantly different between negative and positive HUT results. Especially, HRR/RecSBP and HRR/RecDBP were significantly correlated with induced syncope with a sensitivity and specificity ([60\%, 83\%] cut-off, 0.31; [72\%, 80\%] cut-off, o.63). In the Cox regression, HRR/ RecDBP were significantly associated with recurrence of VVS with hazard ratio of 3.29 (confidence interval, 0.95 to 11.3; $p=0.049$ ).

Conclusions: HRR/RecDBP may be a useful predictor for induction during HUT and recurrence during follow-up in the VVS.

Keywords: Syncope, vasovagal; Exercise test; Tilt-table test

\section{INTRODUCTION}

Vasovagal syncope (VVS) is often problematic condition in young patients [1]. By the age of $60,42 \%$ of females and $32 \%$ of males will have had at least one episode of VVS, and the cumulative incidence and recurrence rate is approximately $25 \%$ to $35 \%$ [2]. In previous report, the number of episodes of syncope is a powerful predictor of recurrence $[3,4]$.

Abrupt changes in hemodynamic status and autonomic function lead to VVS. Most syncopal events can discontinue without any specific therapy, but the reason 
for this is unknown $[1,5]$. Prodromal symptoms and syncopal situation play particularly important roles in the diagnosis of VVS [6].

However, the diagnostic yield by general physician is reported to be $60 \%$ to $70 \%$ with history taking as main factors. In addition, initial assessments did not provide enough evidence for diagnosis due to the stabilization of symptoms after an event [4]. Unpredictable induction of syncopal events also may be embarrassing in the situation of Head-Up table-tilt Test (HUT), and clinical response to such events may be belated. Therefore, additional diagnostic tests with better sensitivity and specificity would be highly desirable [7].

Several studies have evaluated the predictability of syncopal event based on physiological variables [7-9]. Among cardiovascular diagnostic tools, treadmill exercise test (TMT) has been widely used for assessment of important hemodynamic parameters and prognostic implications, such as ischemia, arrhythmias, autonomic function, and exercise-induced symptoms [10,11]. Therefore, our objective was to investigate a simple predictor of induced syncopal episodes during the HUT and recurrence of syncope during clinically follow-up using hemodynamic parameters from TMT in VVS patients.

\section{METHODS}

In total, the 143 consecutive VVS patients were referred by a cardiologist and a neurologist. An initial electrocardiography (ECG), an echocardiogram, and a TMT, all of which were performed prior to, and within 1 week of, HUT from April 2013 to April 2015 in the cohort. This retrospective and prospective cohort of VVS was approved by the Institutional Review Board (201708013) and patient informed consent was obtained at Eulji University Hospital, Daejeon, South Korea. VVS was diagnosed on the basis of a structured history, and clinically suspected if syncope was precipitated by emotional or orthostatic stress, and was accompanied by typical prodromal symptoms, as described in the 2009 European Society of Cardiology (ESC) consensus. VVS is usually classified based on the efferent pathway most involved sympathetic or parasympathetic nerves. The term 'vasodepressor-type VVS' is commonly used if hypotension, due to a loss of upright vasoconstrictor tone, predomi- nates. 'Cardioinhibitory-type VVS' is used when bradycardia or asystole predominate, and 'mixed-type VVS' is used if both mechanisms are present [3]. The neurologist and cardiologist excluded patients who had pre-existing neurologic or cardiovascular disease including transient ischemic attack, seizures, hypertension, coronary artery disease, valvular heart disease, heart failure, atrial fibrillation, diabetes, obesity, and lung disease.

Control subjects, free from syncopal history and without hypertension, diabetic mellitus, coronary artery disease, or lung disease, which were recruited from Eulji Heathcare Center from April 2013 to April 2015 at Eulji University Hospital, Deajeon, South Korea. The TMT data from the 141 controls patients without VVS were considered eligible for analysis in the study.

Two-dimensional and M-mode echocardiography was performed to assess systolic and diastolic function and structure using standard methods [12]. The TMT was performed using GE Healthcare Exercise Stress Test Systems (CASE, GE Medical Systems, Milwaukee, WI, USA) according to the standard Bruce Protocol outlined in Exercise Standards for Testing and Training [10]. ECG (12-lead), heart rate (HR) response, and blood pressure (BP) were recorded at baseline during each stage of exercise, at peak workload, and at 1-minute intervals for 5 minutes during the recovery stage. Results of the TMT were reported by two cardiologists blinded to the patients' backgrounds. The test involved an initial warmup period followed by progressive graded exercise with increasing loads to peak exercise, and then an abrupt post-maximum recovery period. Heart rate recovery (HRR) was defined at the reduction in HR from the peak value to the rate 1 minute later. $\mathrm{BP}$ was measured using a TONOPORT V ambulatory blood pressure system (GE Medical Systems). The peak systolic blood pressure and peak diastolic blood pressure (peakSBP and peakDBP, respectively) during peak workload and the minimum recovery systolic blood pressure and recovery diastolic blood pressure (RecSBP and RecDBP, respectively) during the recovery stage were measured. Measurement time points of HRR, RecSBP and RecDBP was almost same because autonomic nerve activity dramatically changes during recovery phase after exercise.

A positive result of HUT was defined as previously described by the 2009 ESC consensus [3]. Patients were initially placed in supine position for at least 5 minutes 
during which no venous cannulation was performed (pre-tilt phase). Patients were then tilted to, and maintained at, $70^{\circ}$ while being supported by a foot rest for 45 minutes or until syncope. Intravenous isoproterenol was injected in incremental doses ( 1 to $3 \mu \mathrm{g} / \mathrm{min}$ ) to increase HR 25\% from baseline. Recurrent syncopal event was clinically followed by regular telephone contact and out-patient clinic visit.

\section{Statistical analysis}

Baseline characteristics of patients that had positive and negative result of HUT were analyzed and stratified multivariable logistic regression was then constructed to assess the independent relationship for a positive result of HUT. In addition, to determine independent predictors for the recurrent syncope, the multivariate Cox regression hazard model was used. Follow-up variables subjected to univariate screening included the baseline characteristics. Variables showing significant associations with recurrent syncope in the univariate testing were assessed in a multivariate model. A receiver operating characteristic (ROC) curve was plotted to identify the cutoff with optimal sensitivity and specificity for recurrent syncope, and the Kaplan-Myer Curve was plotted for free-from recurrent syncope. Analyses were performed with the MedCalc software version 17.0 (MedCalc Software, Ostend, Belgium). p values < 0.05 were considered to be statistically significant.

\section{RESULTS}

Of the 143 study patients, 92 had negative HUT results and 51 had positive results. The average ages of patients who tested negative HUT, positive HUT and of the controls were $34 \pm 14,29 \pm 11$, and $40 \pm 5$ years, respectively. In the Table 1 , even if, the difference of age between patients that tested positive and negative HUT was slightly different $(p=0.047)$. No differences in body mass index, frequency of syncopal event, prodromal symptom and syncopal situation, or baseline ECG parameters (HR, PR interval, QRS duration, and corrected QT interval) were found between groups. The groups also had similar echocardiographic parameters (ejection fraction, left atrium size and diastolic function parameters). The clinical characteristics and echocardiographic data of the control patients have no abnormal finding due to young healthy people.

Table 1. Baseline clinical characteristics between positive and negative HUT

\begin{tabular}{|c|c|c|c|c|}
\hline Demographic & Total $(\mathrm{n}=143)$ & $\operatorname{HUT}(-)(\mathrm{n}=92)$ & $\operatorname{HUT}(+)(\mathrm{n}=51)$ & $p$ value \\
\hline Age, yr & $31 \pm 19$ & $34 \pm 14$ & $29 \pm 11$ & 0.047 \\
\hline Male sex & $33(23.0)$ & $24(42.9)$ & $9(23.7)$ & 0.056 \\
\hline Body mass index, $\mathrm{kg} / \mathrm{m}^{2}$ & $24.9 \pm 3.0$ & $24.5 \pm 2.9$ & $25 \cdot 4 \pm 3.1$ & 0.532 \\
\hline Prodromal symptom & $107(74.8)$ & $70(76.1)$ & $37(72.5)$ & 0.641 \\
\hline Syncopal situation & & & & 0.401 \\
\hline Walking after standing & & $60(65.2)$ & $28(54 \cdot 9)$ & \\
\hline Long standing or sitting & & $15(16.3)$ & $14(27 \cdot 5)$ & \\
\hline Defecation or micturition & & $9(9.8)$ & $2(3.9)$ & \\
\hline Exertion & & $4(4 \cdot 3)$ & $4(7.8)$ & \\
\hline Stretching & & $2(2.2)$ & $2(3 \cdot 9)$ & \\
\hline Pain or emotional stress & & $2(2.2)$ & $1(2.0)$ & \\
\hline Syncope frequency, no. & $1.1 \pm 1.4$ & $1.1 \pm 1.6$ & $1.1 \pm 1.3$ & 0.548 \\
\hline Heart rate, bpm & $69 \pm 13$ & $69 \pm 13$ & $70 \pm 13$ & 0.677 \\
\hline PR interval, ms & $154 \pm 18$ & $153 \pm 25$ & $156 \pm 23$ & 0.460 \\
\hline QRS duration, ms & $91 \pm 20$ & $91 \pm 22$ & $88 \pm 10$ & 0.379 \\
\hline QTc interval, ms & $421 \pm 23$ & $422 \pm 26$ & $424 \pm 25$ & 0.743 \\
\hline
\end{tabular}

Values are presented as mean $\pm \mathrm{SD}$ or number (\%). HUT, Head-Up table-tilt Test; QTc, corrected QT. 
During the pre-exercise stage, baseline HR, SBP and DBP were similar between patients with negative and positive HUT results; however, during the peak-workload stage, SBP (153 \pm 24 vs. $143 \pm 21)$ and DBP ( $69 \pm 14$ vs. $64 \pm 12)$ were significantly different. During the recovery stage, HRR ( $26 \pm 8$ vs. $33 \pm 10)$, RecSBP $(148 \pm 20$ vs. 132 $\pm 22), \operatorname{RecDBP}(74 \pm 11$ vs. $62 \pm 12), \mathrm{HRR} / \operatorname{RecSBP}(0.18 \pm$ 0.07 vs. $0.25 \pm 0.09$ ), and HRR/RecDBP (0.36 \pm 0.12 vs. 0.53 $\pm 0.19)$ were significant different between control and VVS. In addition, HRR ( $31 \pm 10$ vs. $35 \pm 10)$, RecSBP (134 \pm 22 vs. $127 \pm 22), \operatorname{RecDBP}(63 \pm 12$ vs. $59 \pm 12)$, HRR/RecSBP (0.24 \pm 0.09 vs. $0.28 \pm 0.09)$, and HRR/RecDBP (0.49 \pm 0.18 vs. $0.58 \pm 0.19)$ were significantly different between negative and positive HUT in the Table 2. On the basis of positive result of HUT, HRR, HRR/RecSBP, and HRR/ RecDBP in the mixed type VVS ( $\mathrm{n}=25)$ were significantly different compared to vasodepressor type $(n=10)$ or cardioinhibitory type $\operatorname{VVS}(n=16)$ in the Table 3 .

Logistic regression analysis showed that RecSBP and RecDBP were significantly correlated with positive HUT results. The best ROC curve shows that HRR/RecSBP was a predictor, especially mixed-type VVS, with a sensitivity (60\%), specificity (83\%), cut-off 0.31 , and HRR/
RecDBP also was another predictor, especially mixedtype VVS, with sensitivity (72\%), specificity (80\%), cut-off 0.63 in the induction of syncope during HUT (Fig. 1). In the comparison of ROC curve for recurrent syncope, HRR/RecDBP seems to be more predictable than HRR/ RecSBP in the recurrence of VVS. Especially, HRR/ RecDBP cut-off 0.63 , the hazard ratio of HRR/RecDBP were 3.29 (confidence interval, 0.95 to 11.3; $p=0.049$ ) for recurrence of VVS in the Cox regression during the follow-up (Table 4, Fig. 2).

\section{DISCUSSION}

In this study, patients with VVS had signifcantly different in the RecSBP, RecDBP, HRR/RecSBP, and HRR/ RecDBP during the TMT compared to control patients without VVS. And in addition, the RecSBP, RecDBP, HRR/RecSBP, and HRR/RecDBP during the TMT were significantly different between negative and positive result of HUT. Especially, in the age- and sex-adjusted regression, HRR/RecSBP and HRR/RecDBP were predictable for induction of VVS during HUT and especially

Table 2. Hemodynamic parameters in treadmill exercise test

\begin{tabular}{|c|c|c|c|c|c|c|}
\hline Hemodynamic & Control $(n=141)$ & $\operatorname{VVS}(n=143)$ & $p$ value & $\operatorname{VVS} \operatorname{HUT}(-)(\mathrm{n}=92)$ & $\operatorname{VVS} \operatorname{HUT}(+)(\mathrm{n}=51)$ & $p$ value \\
\hline \multicolumn{7}{|l|}{ Pre-exercise } \\
\hline HR, bpm & $85 \pm 14$ & $88 \pm 14$ & 0.059 & $88 \pm 13$ & $89 \pm 15$ & 0.851 \\
\hline SBP, mmHg & $120 \pm 19$ & $112 \pm 17$ & 0.078 & $113 \pm 16$ & $109 \pm 17$ & 0.218 \\
\hline $\mathrm{DBP}, \mathrm{mmHg}$ & $70 \pm 11$ & $67 \pm 12$ & 0.062 & $68 \pm 12$ & $66 \pm 12$ & 0.423 \\
\hline \multicolumn{7}{|l|}{ Peak-exercise } \\
\hline Workload, METs & $11.7 \pm 1.5$ & $11.4 \pm 1.8$ & 0.175 & $11.3 \pm 2.3$ & $11.5 \pm 1.1$ & 0.099 \\
\hline PeakHR, bpm & $171 \pm 14$ & $175 \pm 15$ & $0.045^{\mathrm{a}}$ & $174 \pm 16$ & $177 \pm 14$ & 0.360 \\
\hline PeakSBP, mmHg & $154 \pm 26$ & $150 \pm 24$ & 0.255 & $153 \pm 24$ & $143 \pm 21$ & $0.009^{a}$ \\
\hline PeakDBP, mmHg & $71 \pm 12$ & $67 \pm 14$ & $0.020^{\mathrm{a}}$ & $69 \pm 14$ & $64 \pm 12$ & $0.044^{\mathrm{a}}$ \\
\hline \multicolumn{7}{|l|}{ Recovery } \\
\hline HRR & $26 \pm 8$ & $33 \pm 10$ & $<0.001^{\mathrm{a}}$ & $31 \pm 10$ & $35 \pm 10$ & $0.044^{\mathrm{a}}$ \\
\hline RecSBP, mmHg & $148 \pm 20$ & $132 \pm 22$ & $<0.001^{\mathrm{a}}$ & $134 \pm 22$ & $127 \pm 22$ & 0.066 \\
\hline RecDBP, mmHg & $74 \pm 11$ & $62 \pm 12$ & $<0.001^{\mathrm{a}}$ & $63 \pm 12$ & $59 \pm 12$ & 0.090 \\
\hline HRR/RecSBP & $0.18 \pm 0.07$ & $0.25 \pm 0.09$ & $<0.001^{\mathrm{a}}$ & $0.24 \pm 0.09$ & $0.28 \pm 0.09$ & $<0.001^{\mathrm{a}}$ \\
\hline HRR/RecDBP & $0.36 \pm 0.12$ & $0.53 \pm 0.19$ & $<0.001^{\mathrm{a}}$ & $0.49 \pm 0.18$ & $0.58 \pm 0.19$ & $0.008^{a}$ \\
\hline
\end{tabular}

Values are presented as mean $\pm \mathrm{SD}$.

VVS, vasovagal syncope; HUT, Head-Up table-tilt Test; HR, heart rate; SBP, systolic blood pressure; DBP, diastolic blood pressure; MET, metabolic equivalent; HRR, heart rate recovery; Rec, recovery stage.

a $p<0.05$ statistically significant. 
Table 3. Hemodynamic parameters of positive HUT in treadmill exercise test

\begin{tabular}{lcccc}
\hline Hemodynamic & \multicolumn{5}{c}{ VVS } & $\begin{array}{c}p \\
\text { value }\end{array}$ \\
\cline { 2 - 4 } Number & VD & CI & Mixed & \\
Age, yr & 10 & 16 & 25 & \\
Male sex & $5(50.0)$ & $7(43.7)$ & $5(20.0)$ & 0.139 \\
Pre-exercise & & & & \\
HR, bpm & $87 \pm 12$ & $89 \pm 15$ & $88 \pm 16$ & 0.755 \\
SBP, mmHg & $110 \pm 13$ & $114 \pm 19$ & $107 \pm 18$ & 0.759 \\
DBP, mmHg & $69 \pm 9$ & $71 \pm 15$ & $62 \pm 11$ & 0.184 \\
Peak-exercise & & & & \\
Workload, & $11.7 \pm 1.2$ & $12.1 \pm 1.9$ & $11.5 \pm 1.1$ & 0.217 \\
METs & & & & \\
HR, bpm & $166 \pm 20$ & $176 \pm 16$ & $175 \pm 14$ & 0.529 \\
\hline SBP, mmHg & $141 \pm 11$ & $152 \pm 24$ & $139 \pm 20$ & 0.378 \\
\hline DBP, mmHg & $69 \pm 12$ & $66 \pm 13$ & $61 \pm 12$ & 0.590 \\
\hline Recovery & & & & \\
\hline HRR & $30 \pm 10$ & $33 \pm 9$ & $39 \pm 9$ & 0.057 \\
\hline SBP, mmHg & $116 \pm 7$ & $133 \pm 24$ & $128 \pm 21$ & 0.601 \\
\hline DBP, mmHg & $58 \pm 9$ & $63 \pm 12$ & $58 \pm 12$ & 0.263 \\
\hline HRR/SBP & $0.27 \pm 0.11$ & $0.25 \pm 0.08$ & $0.30 \pm 0.07$ & $0.047^{\mathrm{a}}$ \\
\hline HRR/DBP & $0.51 \pm 0.19$ & $0.52 \pm 0.24$ & $0.65 \pm 0.13$ & $0.037^{\mathrm{a}}$ \\
\hline
\end{tabular}

Values are presented as mean $\pm \mathrm{SD}$.

HUT, Head-Up table-tilt Test; VVS, vasovagal syncope; VD, vasodepressor type; CI, cardioinhibitory type; HR, heart rate; SBP, systolic blood pressure; DBP, diastolic blood pressure; MET, metabolic equivalent; HRR, heart rate recovery. ${ }^{a} p<0.05$ statistically significant.

HRR/RecDBP was significantly associated with recurrent syncope during follow-up period.

VVS was considered as cardiovascular reflexes that are normally maintained in controlling the circulation become intermittently inappropriate, in response to a trigger, resulting in vasodilatation and, or bradycardia and thereby in a fall in arterial BP and global cerebral perfusion [3]. Therefore, HUT is the standard tool used to diagnose and establish autonomic changes in patients with VVS [7]. HUT also has an area under the ROC curve of 0.84 and an adjusted diagnostic odds ratio of 12.15 for VVS [13]. Therefore, many physicians believe that HUT, when interpreted appropriately, has diagnostic value. In particular, induction of VVS symptoms during the HUT can be an important end point of examination. How-

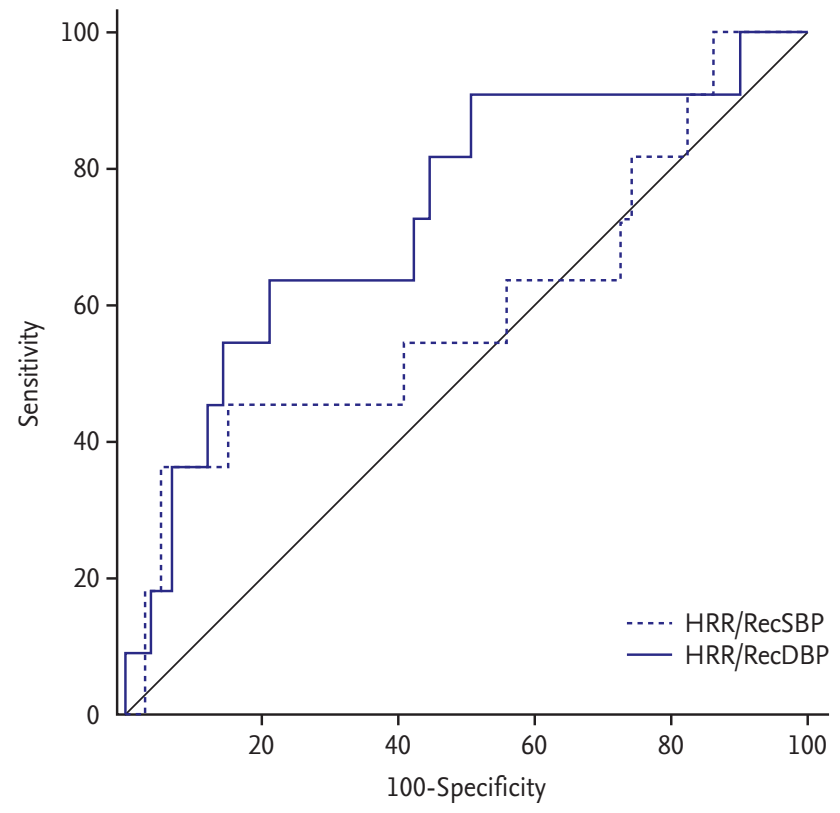

Figure 1. Receiver operating characteristic presenting predictive ability of heart rate recovery (HRR)/recovery systolic blood pressure (RecSBP) and HRR/recovery diastolic blood pressure (RecDBP) of induced syncopal episode during Head-Up table-tilt Test.

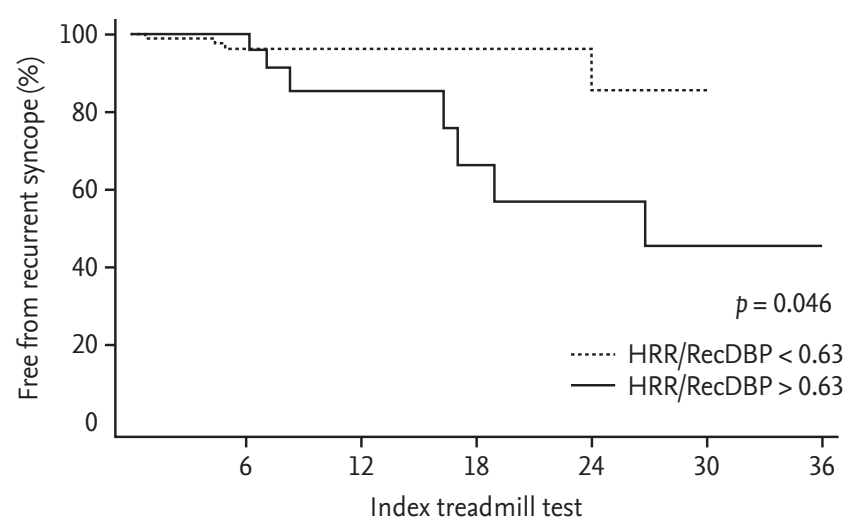

Number at risk

Group: $\mathrm{HRR} / \operatorname{RecDBP}<0.63$

$\begin{array}{ccccccc}103 & 52 & 18 & 11 & 8 & 0 & 0 \\ \text { Group: } \mathrm{HRR} / \operatorname{RecDBP}>0.63 & & & & & \\ 40 & 25 & 12 & 7 & 5 & 1 & 1\end{array}$

Figure 2. Comparison of receiver operating characteristic presenting predictive ability of heart rate recovery (HRR)/ recovery systolic blood pressure (RecSBP) and HRR/recovery diastolic blood pressure (RecDBP) of recurrent syncopal episode during follow-up. Kaplan-Meier curve presenting predictive ability of HRR/RecDBP for recurrent syncopal episode during follow-up.

ever, there is uncertainty regarding the length of time required for the test to be an accurate diagnostic or pre- 
Table 4. Logistic regression of prediction for positive HUT

\begin{tabular}{|c|c|c|c|c|c|c|}
\hline \multirow{2}{*}{ Variable } & \multicolumn{3}{|c|}{ Univariate } & \multicolumn{3}{|c|}{ Age-/sex-adjusted } \\
\hline & OR & $95 \% \mathrm{CI}$ & $p$ value & OR & $95 \% \mathrm{CI}$ & $p$ value \\
\hline Age & 0.97 & $0.93-1.00$ & 0.074 & & & \\
\hline Sex & 2.41 & $0.96-6.04$ & 0.059 & & & \\
\hline PeakSBP & 0.98 & $0.96-1.00$ & 0.164 & & & \\
\hline PeakDBP & 0.96 & $0.93-1.00$ & 0.037 & & & \\
\hline HRR & 1.05 & $1.00-1.09$ & 0.019 & 1.05 & $1.00-1.10$ & 0.036 \\
\hline RecSBP & 0.97 & $0.95-0.99$ & $0.008^{\mathrm{a}}$ & & & \\
\hline RecDBP & 0.95 & $0.92-0.99$ & $0.025^{\mathrm{a}}$ & & & \\
\hline HRR/RecSBP & $105 \cdot 52$ & $2.23-498.9$ & $0.013^{\mathrm{a}}$ & 128.3 & $2.43-675.8$ & $0.007^{\mathrm{a}}$ \\
\hline HRR/RecDBP & 11.43 & $1.76-74.28$ & $0.007^{\mathrm{a}}$ & 8.02 & $1.16-55.10$ & $0.014^{\mathrm{a}}$ \\
\hline \multicolumn{7}{|l|}{ Mixed type VVS } \\
\hline HRR/RecSBP & 56.05 & $5.82-539.4$ & $0.006^{a}$ & $77 \cdot 6$ & $2.60-127.2$ & $0.010^{\mathrm{a}}$ \\
\hline HRR/RecDBP & 51.83 & $4.80-559.2$ & $0.001^{a}$ & 29.50 & $2.56-339.5$ & $0.006^{\mathrm{a}}$ \\
\hline
\end{tabular}

HUT, Head-Up table-tilt Test; OR, odds ratio; CI, confidence interval; SBP, systolic blood pressure; DBP, diastolic blood pressure; HRR, heart rate recovery; Rec, recovery stage; VVS, vasovagal syncope.

${ }^{\mathrm{a}} \mathrm{p}<0.05$.

dictive tool (Supplementary Table 1) [14].

Induction of VVS during HUT are also embarrassing and can lead to patients delaying treatment, especially if the physicians and medical personnel involved are not highly trained to deal with the situation. However, the examination of HUT could be more easily available if there offer a simple way to predict induction of VVS.

Sheldon et al. [15] were the first to demonstrate a correlation between recurrent syncope and positive results of HUT. The most powerful predictor of recurrent syncope was the logarithm of the number of preceding syncopal events [15]. In addition, Baron-Esquivias et al. [1] also analyzed significant differences in the rate of recurrent syncope between patients with $<5$ or $\geq 5$ previous episodes of syncope ( $25 \%$ vs. $44 \%)$. Aydin et al. [9] also demonstrated that the number of previous syncopal events, history of bronchial asthma, and female gender were predictors for recurrent syncope. In our study, all patients had less than five previous syncopal events, no pulmonary disease, and these factors should not influence the results.

HUT has been considered as non-invasive methods to measure autonomic modulation from $\mathrm{HR}$ and $\mathrm{BP}$ variability, and have been used in several clinical applications as physiological markers of cardiac autonomic control [16-18]. An initial increase in neuroendocrine sympathetic activity is followed by an increase in vagally-mediated parasympathetic activity with a concurrent withdrawal of sympathetic activity. Previous studies have shown that patients with VVS have dysfunctional baroreflex regulation during orthostatic stress, abnormal sensitivity of pressure receptors in the heart and arterial system, abnormalities of gain in central nervous system processing, and failure of the normal pressure regulatory mechanism compared to patients without VVS [19]. In our cohort, most of recurrent VVS as referral center consist of predominantly young female which may be explained by more over-reactive and unbalanced efferent pathway involved sympathetic or parasympathetic nerves.

We assumed that BP and HR in the TMT may reflect sympathetic and parasympathetic nerve activity in VVS patients. Evaluation of the HR response during the TMT is easy to perform and considered a useful technique that reflects autonomic activity changes [20]. HRR reflects the rate of HR decline after cessation of exercise, and is defined as a HR reduction between maximal HR during peak-exercise and HR during the recovery stage $[21,22]$. The first minute HRR curve is a novel concept in exercise physiology and reflects sympathetic withdrawal and vagal augmentation [23]. The withdrawal of sympathetic tone to the veins, which results in peripheral 
blood pooling, has been suggested to be an important factor contributing to the decrease in cardiac output that occurs during VVS [24]. Vasodilatation in other vascular beds, including splanchnic and renal circulation, could also occur early in the development of syncope. The transduction of sympathetic outflow into peripheral vascular resistance may also be altered before sympathetic withdrawal $[25,26]$, and this may be why hemodynamic parameters changes differed in control patients compared to VVS patients in our study. Since HRR, SBP and DBP are controlled by the baroreflex response during the recovery stage, we assumed that a combination of their variables (HRR/RecSBP and HRR/RecDBP) improve their predictive power more than single variable, HRR, RecSBP, and RecDBP in the induction and recurrence of VVS, especially mixed-type VVS, compare to vasodepressor or cardioinhibitory-type.

RecDBP seems to be more decreased in patients with positive HUT compared to negative HUT in the Table 2 because RecDBP could be more influenced by peripheral vascular resistance. A significant reduction of RecDBP may reflect the possible loss of vasoconstriction correlated with withdrawal of sympathetic tone prior to syncope [24-26].

The hemodynamic index proposed by using the TMT seemed to be better predicted than number of previous syncopal events, and female gender which can serve as a surrogate diagnostic tool in clinical practice to manage VVS. According to the 2015 Heart Rhythm Syncope Consensus [4], there is growing movement away from HUT, particularly because it is not either incrementally useful to a structured history or predictive of outcome. Much of this is borne out in this study where the positive and negative result of HUT is similar in almost all of the relevant history and clinical parameters.

The unpredictable induction during HUT and recurrence of syncopal events during clinically follow-up in the real practice may be embarrassing to examiner and physician and could take time to deal with the unexpected situation and traumatic events. The clinical implication of the study is that the physician be recommended to take more preparation and prompt care of induction of VVS patients during the HUT and recurrence of VVS during follow-up period according to simple parameters of TMT in advance.

Our study should be interpreted in the context of the following limitations. First, the proportion of our study was more female than male patients in the VVS. Therefore, the age-, sex-adjusted analysis could be statistically reasonable for our result. Second, HUT seems to be more common than TMT as a clinical examination for patients with VVS, potential confounding factors may exist despite our efforts to adjust for differences; however, this occurs in most cohort studies. Third, in our facility, TMT was performed in almost patients with VVS. However, there exist selection biases. Fourth, the patients with VVS were classified by a single HUT result. The reproducibility of HUT in the patients with VVS should be reported.

In conclusion, HRR/RecSBP and HRR/RecDBP, taken from the TMT, may be a useful predictor of induced syncope during HUT. Especially, HRR/RecDBP was significantly associated with recurrent syncope during clinically follow-up.

\section{KEY MESSAGE}

1. The recurrence of vasovagal syncope is still unpredicted.

2. The heart rate and blood pressure during the recovery stage of treadmill exercise test was significantly associated with recurrence of vasovagal syncope.

3. The heart rate recovery/recovery diastolic blood pressure may be a useful predictor of induced vasovagal syncope during Head-Up table-tilt Test and recurred vasovagal syncope during the follow-up.

\section{Conflict of interest}

No potential conflict of interest relevant to this article was reported.

\section{Acknowledgments}

This research was supported by Grants 2017 EMBRI DJooo4 from the Eulji University.

\section{REFERENCES}

1. Baron-Esquivias G, Errazquin F, Pedrote A, et al. Long- 
term outcome of patients with vasovagal syncope. Am Heart J 2004;147:883-889.

2. Cintra F, Poyares D, DO Amaral A, et al. Heart rate variability during sleep in patients with vasovagal syncope. Pacing Clin Electrophysiol 2005;28:1310-1316.

3. Task Force for the Diagnosis and Management of Syncope; European Society of Cardiology (ESC); European Heart Rhythm Association (EHRA), et al. Guidelines for the diagnosis and management of syncope (version 2009). Eur Heart J 2009;30:2631-2671.

4. Sheldon RS, Grubb BP 2nd, Olshansky B, et al. 2015 Heart Rhythm Society expert consensus statement on the diagnosis and treatment of postural tachycardia syndrome, inappropriate sinus tachycardia, and vasovagal syncope. Heart Rhythm 2015;12:e41-e63.

5. D'Ascenzo F, Biondi-Zoccai G, Reed MJ, et al. Incidence, etiology and predictors of adverse outcomes in 43,315 patients presenting to the Emergency Department with syncope: an international meta-analysis. Int J Cardiol 2013;167:57-62.

6. Guida P, Iacoviello M, Forleo C, et al. Prevalence, timing, and haemodynamic correlates of prodromes in patients with vasovagal syncope induced by head-up tilt test. Europace 2009;11:1221-1226.

7. Grubb BP. Clinical practice. Neurocardiogenic syncope. N Engl J Med 2005;352:1004-1010.

8. Mereu R, De Barbieri G, Perrone T, Mugellini A, Di Toro A, Bernardi L. Heart rate/blood pressure ratio as predictor of neuromediated syncope. Int J Cardiol 2013;167:11701175 .

9. Aydin MA, Maas R, Mortensen K, et al. Predicting recurrence of vasovagal syncope: a simple risk score for the clinical routine. J Cardiovasc Electrophysiol 2009;20:416421.

10. Fletcher GF, Ades PA, Kligfield P, et al. Exercise standards for testing and training: a scientific statement from the American Heart Association. Circulation 2013;128:873-934.

11. Shin JH, Kang KW, Kim JH, et al. Treadmill exercise-induced E/e' elevation as a predictor of cardiovascular event in end-stage renal disease on peritoneal dialysis. Korean J Intern Med 2016 Nov 11 [Epub]. https://doi.org/10.3904/ kjim.2016.254.

12. Mor-Avi V, Lang RM, Badano LP, et al. Current and evolving echocardiographic techniques for the quantitative evaluation of cardiac mechanics: ASE/EAE consensus statement on methodology and indications endorsed by the Japanese Society of Echocardiography. J Am Soc Echocardiogr 2011;24:277-313.

13. Forleo C, Guida P, Iacoviello M, et al. Head-up tilt testing for diagnosing vasovagal syncope: a meta-analysis. Int J Cardiol 2013;169:e49-e50.

14. Aerts AJ, Dendale P, Block P, Dassen WR. Reproducibility of nitrate-stimulated tilt testing in patients with suspected vasovagal syncope and a healthy control group. Am Heart J 2005;150:251-256.

15. Sheldon R, Rose S, Flanagan P, Koshman ML, Killam S. Risk factors for syncope recurrence after a positive tilt-table test in patients with syncope. Circulation 1996;93:973981.

16. Virag N, Sutton R, Vetter R, Markowitz T, Erickson M. Prediction of vasovagal syncope from heart rate and blood pressure trend and variability: experience in 1,155 patients. Heart Rhythm 2007;4:1375-1382.

17. Shinohara T, Ebata Y, Ayabe R, et al. Cardiac autonomic dysfunction in patients with head-up tilt test-induced vasovagal syncope. Pacing Clin Electrophysiol 2014;37:16941701.

18. Zygmunt A, Stanczyk J. Heart rate variability in children with neurocardiogenic syncope. Clin Auton Res 2004;14:99-106.

19. Bechir M, Binggeli C, Corti R, et al. Dysfunctional baroreflex regulation of sympathetic nerve activity in patients with vasovagal syncope. Circulation 2003;107:1620-1625.

20. Cole CR, Blackstone EH, Pashkow FJ, Snader CE, Lauer MS. Heart-rate recovery immediately after exercise as a predictor of mortality. N Engl J Med 1999;341:1351-1357.

21. Nishime EO, Cole CR, Blackstone EH, Pashkow FJ, Lauer MS. Heart rate recovery and treadmill exercise score as predictors of mortality in patients referred for exercise ECG. JAMA 2000;284:1392-1398.

22. Morshedi-Meibodi A, Larson MG, Levy D, O'Donnell CJ, Vasan RS. Heart rate recovery after treadmill exercise testing and risk of cardiovascular disease events (The Framingham Heart Study). Am J Cardiol 2002;90:848-852.

23. Okutucu S, Karakulak UN, Aytemir K, Oto A. Heart rate recovery: a practical clinical indicator of abnormal cardiac autonomic function. Expert Rev Cardiovasc Ther 2011; 9:1417-1430.

24. Epstein SE, Stampfer M, Beiser GD. Role of the capacitance and resistance vessels in vasovagal syncope. Circulation 1968;37:524-533.

25. Cooke WH, Rickards CA, Ryan KL, Kuusela TA, Converti- 
no VA. Muscle sympathetic nerve activity during intense lower body negative pressure to presyncope in humans. J Physiol 2009;587(Pt 20):4987-4999.
26. Fu Q, Levine BD. Pathophysiology of neurally mediated syncope: role of cardiac output and total peripheral resistance. Auton Neurosci 2014;184:24-26. 
Supplementary Table 1. Recurrence of vasovagal syncope between negative and positive HUT

\begin{tabular}{lcccc}
\hline Variable & Total $(\mathrm{n}=143)$ & HUT $(-)(\mathrm{n}=92)$ & HUT $(+)(\mathrm{n}=51)$ & $p$ value \\
\hline Recurrent syncope & $11(7.6)$ & $8(8.6)$ & $3(5.8)$ & 0.546 \\
Follow-up duration, mon & $8.6 \pm 8.0$ & $8.8 \pm 7.8$ & $8.4 \pm 8.5$ & 0.789 \\
\hline
\end{tabular}

Values are presented as number (\%) or mean \pm SD.

HUT, Head-Up table-tilt Test. 